\title{
Extracting Semantic Roles from a Model of Eventualities
}

\author{
Sylvie Ratté \\ Université du Québec à Montréal / Linguistics Department \\ C.P. 8888, Succ. "A" / Montréal, QC / H3C 3P8 \\ e-mail: sr@info.uqam.ca
}

The notion of semantic roles is usually attributed to Fillmore [8], however its history can be traced back through Tesnière [16] to Panini. Following this tradition, many researchers recognize their usefulness in the description of language - even if they do not agree on their significance [7]. However, a weak or strong commitment to this notion does not elude the fact that it proves to be very difficult to settle on a finite set of labels along with their formal definitions. The dilemma resulting from this challenge is well known: to require a univocal identification by each role results in an increase in their number while to abstract their semantic content gives rise to an inconsistent set. If a finite set is possible, one has to find a proper balance between these two extremes. As a result, every flavor of roles have been used from time to time in linguistics (e.g., GB, in the spirit of Fillmore, HPSG, in the line of situation semantics), and also in AI [10, see also 4].

Between the total refusal to use those labels (as in GPSG) and the acceptance of individual roles (as in HPSG) there is a wide range of proposals on what constitute a good set of L(inguistic)-Roles [7] and, as a consequence, on the way to differentiate between them and define them. Most of the definitions have been based on the referential properties that can be associated with each role bearer (e.g. an AGENT is a volitional animate entity). Even if this approach is necessary at one time or another, this kind of definition inevitably leads to either the "let's create another role" or the "let's abstract its definition" syndromes. Properties are not always of the static kind though. Sometimes, dynamic properties are also used (e.g. an AGENT is the perceived instigator of the action).

Since one of the desired characteristic of a roles system is the power to discriminate events [5] (another "desired" property being to offer an easier selection of grammatical functions), the recognition of semantic roles should be linked to the interpretation of the event, that is to their dynamic properties. In a study on locative verbs in French, Boons [3] has convincingly shown the importance of taking into account aspectual criteria in the description of a process, suggesting that GOAL and SOURCE roles should be reinvestigated in the light of those criteria. It is our hypothesis that proliferation of roles is a natural phenomenon caused by the specialized properties required by the interpretation of a predicate within a specific semantic field: to overlook these properties yields the over-generalization already mentionned. The best way to approach the expansion/contraction dilemma is to search for the minimal relations required for a dynamic interpretation of events (in terms of their aspectual criteria and through an identification of all the participants in it).

Our first step toward this abstraction was to consider each participant (individuals or properties) either as a localized entity (a token) or a location (a place), and to determine its role in the realization of the process expressed by the predicate. The model exhibits some common points with a localist approach $[1,11]$ since it recognizes (in an abstract sense) the importance of spatio-temporal "regions" in the process of individuation of events [14]. To express the change of localization (again in an abstract sense), the notion of transitions is used. The entire construction is inspired by Petri net theory [15]: a set $S$ of places, a set $T$ of transitions, a flow relation $F:(S \times T) \rightarrow(T \times S)$ and markers are the categories used to define the structure of a process (and as a consequence of the events composing it).

For example, the dynamic representation of Max embarque la caisse sur le cargo [3]/Max embarks the crate on the cargo boat can be analyzed in two steps. First there is a transition from an initial state IS where the crate is not on the cargo boat to a final state FS where the crate is on the cargo boat. The final state can be expressed by the static passive, la caisse est embarquée sur le cargolthe crate was embarked on the cargo boat, and is schematized in (2). One of the argument (cargo boat) is used as a localization while the other argument is used as a localized entity (crate), the THEME according to Gruber [9]. The initial state can be expressed (in this case) by the negation of the final state and is schematized in (1). The realization of the entire process is then represented by the firing of the net which can be illustrated by the snapshots (1) and (2).
1. Is: $O \rightarrow \vdash \circ: \mathrm{FS}$
2. Is: $\mathrm{O}-1-\mathrm{O}: \mathrm{FS}$
To integrate the participation of "Max" in the model, we recognize the importance of 
causality in the discrimination of events $[13,14]$. Since the cause is understood to be the first entity responsible for the realization of events [6], the obvious schematization is (3).

\section{3. $\mathrm{O} \rightarrow \mathrm{1} \rightarrow \mathrm{O} \rightarrow \mathrm{H}$ 4. $\mathrm{O}-1-\mathrm{O}-\mathrm{-O}$}

It is possible that a recursive definition (places and transitions) will be necessary to express "properly" the causation, the localization of events and processes or the concept of dynamic states $[2,14]$. In that case, the schematization could then be (4). But we can achieve the same result through a proper type definition of the transition expressing the cause: $(s \times t) \rightarrow(t \times$ $((s \times t) \rightarrow(t \times s)))$, where " $s$ " is a place and " $t$ ", $a$ transition.

This approach to semantic roles determination is close to the one undertook by Jackendoff [12]. His identification of each role to a particular argument position in a conceptual relation is given here by the way it participate to the firing of the net. (It is our guess that most of the conceptual relations used by Jackendoff can be expressed within this model, giving to them an operational interpretation.) The model has the advantage to give an explicit and simple definition of relations that do not have the same semantic range (e.g. CAUSE vs FROM vs AT).

The analysis of locative processes using abstract regions instead of the traditional roles is better because it is, we think, the real basis of those interpretations. Abstracting away referential properties gives the basic interactions expressed by the predicate. Specifying those properties within a specific semantic field gives rise to the set of roles we are used to (e.g. within the spatial field, schematizations (1) and (2) express SOURCE and GOAL roles).

With this model we were able to give an operational description of the difference between Max charge des briques dans le camion/Max loads bricks in the truck and Max charge le camion de briques/Max loads the truck with bricks. The schematization take into account which participant is responsible for each transition firing and thus can lead us to the "final" place. As a first approximation of these continuous processes, (5) and (6) are proposed (the direct contribution of the instrument is also introduced). But recognition, as a participant of the quantity of bricks in (5) and the capacity of the truck in (6), results in the schematizations (7) et (8) (both display a specialization of their direct object in order to complete the semantic interpretation).

\section{5.}

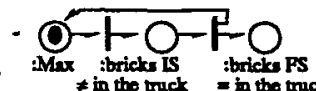

6.

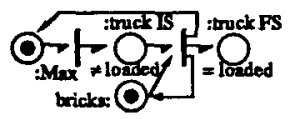

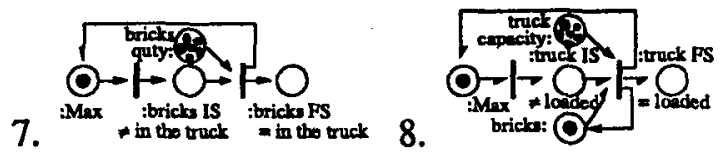

By its simplicity, the model can thus give rise to "confusion" over some roles, in accordance with the general tendancy to observe "roles clusters". The resulting notation seems also an interesting way to explore the differences between static and dynamic processes, differences that are not very "visual" if one is using a static notation.

Our research is now directed toward the analysis of the system when more semantic content is used. We are testing if these adds-on have impacts on its behaviour, while analyzing if the partial semantic interpretation gives rise to the predicted syntactic forms (that is how does each potential participant is grammaticalized).

\section{References}

[1] Anderson, J.M., 1971. The grammar of case, Towards a localistic theory, CUP: Cambridge.

[2] Bach, E. 1986. The Algebra of Events, Linguistics and Philosophy 9:5-16.

[3] Boons, J.-P., 1987. La notion sémantique de déplacement dans une classification syntaxique des verbes locatifs. Langue française 76, Dec: 5-40.

[4] Bruce, B., 1975. Case Systems for Natural Language. Artificial Intelligence 6, 327-360.

[5] Carlson, G., 1984. Thematic roles and their role in semantic interpretation. Linguistics 22: 259-279.

[6] Delancey, S., 1984. Notes on Agentivity and Causation. Studies in Language, 8.2: 181-213.

[7] Dowty, D. R., 1989. On the Semantic Content of the Notion of "Thematic Role", in Properties, Types and Meaning, II. G. Chierchia, B. H. Partee, \& R. Tumer (eds), Kluwer: Boston, 69-129.

[8] Fillmore, C. J., 1968. The Case for Case, in Universals in Linguistic Theory. Bach \& Harms (eds), Holt, Rinehart \& Winston: New York, 1-88.

[9] Gruber, J., 1976. Lexical structures in syntax and semantics. North-Holland: New York.

[10] Hirst, G., 1987. Semantic interpretation and the resolution of ambiguity. CUP: New York.

[11] Hjemslev, L., 1972. La catégorie des cas, Wilhem Fink Verlag München: Band, (1935-1937).

[12] Jackendoff, R. S., 1990. Semantic Structures.

MIT Press: Cambridge MA

[13] Michotte, A. E., 1954. La perception de la causalité. Pub. univ. de Louvain, Erasme S.A.: Paris. [14] Miller, G. A. and P.N. Johnson-Laird, 1976.

Language and Perception. Belknap Press of Harvard University Press: Cambridge MA.

[15] Reisig, W. 1985. Petri Nets, An Introduction. Springer-Verlag: New York.

[16] Tesnière, L., 1959. Eléments de Syntaxe Structurale. Klincksieck: Paris. 